\title{
Historia especulativa del presente
}

\section{Speculative History of the Present}

\author{
Claudio Canaparo \\ Birkbeck College (Reino Unido) \\ c.canaparo@sllc.bbk.ac.uk
}

\section{Resumen}

En este artículo se examina de qué modo ha afectado a la idea de historia del presente la desaparición del historicismo decimonónico o concepción en la que filosofía y de lo histórico giraban exclusivamente en torno a la categoría de temporalidad. A este respecto se insiste en tres consecuencias: primera, una ampliación del debate sobre qué es lo histórico hacia el tema de cuáles son los procedimientos por los que decidimos lo que es historiable; segunda, una concepción del presente en la cual este deja de ser una mera colección de eventos relevantes y se convierte en una construcción intelectual; y tercero, cómo el presente deja de identificarse simplemente con lo inmediato.

\section{Palabras clave}

Presente, construcción, conceptos, actividad especulativa.

\begin{abstract}
This article examines how the disappearance of the nineteenth-century historicism, or conception where philosophy and the historic hinged exclusively on the category of temporality, affects the idea of the history of the present. In this respect, three consequences are to insist on: first, an enlargement of the debate on what the historic is towards the topic of what are the procedures through which we decide what is historical and what not. The second consequence is a conception of the present where this ceases to be a simple collection of relevant events to become an intellectual construction. The third aspect is how the present no longer identifies simply with the immediate.
\end{abstract}

\section{Key Words}

Present, construction, concepts, speculative activity. 


\section{Introducción}

Para no pocos historiadores la historia del presente es imposible por su propia definición, por cuanto habría una discontinuidad natural entre presente e historia, de lo cual se deduce que el historiador no se ocuparía de elementos del presente. Esta perspectiva adquiriría sentido para aquellos que definen el mundo a partir de su historicidad, es decir, a partir de un principio de temporalidad. Con método similar, aunque con perspectiva opuesta, otros historiadores sostienen que el presente es la historia en estado puro por cuanto los sucesos y eventos del ahora y el mientras tanto determinan aquello que pensamos del pasado y el significado futuro de este ahora $\mathrm{y}$ mientras tanto cuando adquiera la categoría de presente sucedido. En este último caso la historia del presente es definida a menudo menos por una problemática epistemológica que por el contenido actual de sus argumentos, razón por la cual algunos autores fusionan historia y ciencia política. ${ }^{1}$

Más allá de estas dos perspectivas, el hecho que los historiadores no se ocupen de elementos del presente tiene que ver en la actualidad menos con una elección epistemológica que con una realidad de las dinámicas financieras y tecnológicas, las cuales escapan a un planteamiento temporal de tipo clásico. Los historiadores, por el contrario, sí se han visto obligados a ocuparse del estatuto conceptual y epistemológico del presente en cuanto tal. ${ }^{2}$

Por otra parte, a esta coincidencia de ambas posturas indicadas antes en torno a la categorización del presente como problemático es a aquello que se conoció a partir del siglo XIX, sobre todo por razones argumentativas, como un exceso de historia, como un sobrecarga de elementos en torno a la necesidad de justificar el ahora y mientras tanto en una estructura temporal. A esta perspectiva es a lo que se ha denominado historicismo, que es en definitiva una formulación que piensa el mundo a partir de una noción de temporalidad - lo cual, no por casualidad, coincidía así con la tradición filosófica clásica- y con la historia de la misma -que concebían al tiempo como la cuestión filosófica, en relación con lo humano, más fundamental-. La relación de la filosofía con la historia de la filosofía se convirtió así en un modelo histórico sobre el cual unos y otros encontraban recursos para acercar o alejar el presente. The Idea of History (1946) de R. G. Collingwood (1889-1943) es un ejemplo consumado de esta situación.

Cuando esta situación especulativa fue modificada y la noción de tiempo ya no constituyó un motor especulativo central, el historicismo perdió su sustento epistemológico más eficaz. ${ }^{3}$ El agotamiento de la centralidad de la historia de la filosofía en la concepción de una noción de especulación filosófica es también el fin del historicismo como única manera de concebir lo histórico humano. Contrariamente a lo

\footnotetext{
1 Véase por ejemplo Pierre Rosanvallon, Le Peuple introuvable: Histoire de la représentation démocratique en France (Paris: Gallimard, 1998), y Pierre Rosanvallon, La Démocratie inachevée. Histoire de la souveraineté du peuple en France (Paris: Gallimard, 2000).

${ }^{2}$ Paul Veyne, Comment on écrit l'histoire. Essai d'épistémologie (Paris: Seuil, 1971).

${ }^{3}$ Véase por ejemplo Philippe Ariès, L'Homme devant la mort (Paris: Seuil, 1977) y Philippe Ariès, Le Temps de l'histoire (Éditions du Rocher: Paris, 1986); y sobre todo Paul Veyne, Comment on écrit l'histoire. Essai d'épistémologie (Paris: Seuil, 1971).
} 
que piensan ciertos autores, aquello que representa una cuestión en la actualidad es menos el estatuto de lo histórico como la discusión cerca de lo historiable, es decir, no solo acerca de qué cosa es histórica, sino también acerca de qué método, de qué manera, decidimos qué va a ser historia y qué simplemente información. Y esta cuestión clásica tiene sin embargo una connotación inesperada. ${ }^{4}$ En términos clásicos aquello que no era historia pasaba al olvido, de alguna manera desaparecía o sobrevivía malamente como leyenda o ruina. De modo que información y narración histórica coincidían o, mejor dicho, la legitimidad de lo histórico en gran medida residía en una especie de selección informativa e institucional. Por el contrario, en la actualidad, lo historiable está obligado a convivir con la información y con la basura o los residuos, que son las formas actuales de ruinas, de manera que lo historiable no será, a diferencia de lo que sucedía en el pasado, la única fuente legítima de lo pasado humano.

En este sentido es interesante observar cómo se ha confundido el debate en torno a (i) el estatuto epistémico y conceptual del presente, ${ }^{5}$ con (ii) la finalidad en sentido aristotélico de lo historiable, ${ }^{6}$ y (iii) con la pertinencia filosófica de pensar el hecho humano y su evolución a partir de un planteamiento temporal. ${ }^{7}$ Que todavía se produzcan este tipo de confusiones generalizadas prueba que el paradigma conceptual decimonónico de la filosofía y la historia europeas persiste en la manera que se piensan las cuestiones acerca de aquello que consideramos como historiable y de su relación a una idea de presente.

La noción de presente entonces se ha visto modificada no solo, como decimos, cuando la temporalidad ya no constituye el centro de gravedad de la reflexión histórica o filosófica, sino además cuando el presente comienza a ser concebido como una construcción y, por consecuencia, las narraciones históricas comienzan a desarrollarse a partir de horizontes de expectativa y de construcciones mediáticas de dicho presente. Bajo estas condiciones, lo histórico en el presente ya no es definido por hechos o eventos relevantes $-\mathrm{o}$ por instituciones- sino por conceptualizaciones vinculadas a cuestiones que podríamos llamar sociológicas: es la concepción de presente -una dimensión tecnológica, una dimensión especulativa, una dimensión conceptual- aquello que determina el presente en cuanto materia historiable. Algunos historiadores piensan que esta situación es un anuncio del "fin de la historia", por el contrario, una cuestión de este género nos empuja al centro mismo de discusión acerca de lo histórico en términos clásicos, como ejemplo, en torno al sentido de akribeia. ${ }^{8}$ Pero, sobre todo, nos fuerza a pensar acerca del sentido de las trazas, de las ruinas, de la información y de la selección de los materiales y aparatos que no pasarán al olvido. Estamos viviendo, por primera vez en la cultura Occidental, un darwinismo histórico sin precedentes cuya deriva

\footnotetext{
${ }^{4}$ Véase por ejemplo Theodor Mommsen, Rome: from earliest times to 44 B. C. (London: Collier and Sons, 1913) y Edward Gibbon, The History of the Decline and Fall of the Roman Empire (New York: Penguin Books, 2000 [1776-1789]).

${ }^{5}$ Véase por ejemplo Paul Ricoeur, Temps et récit (Paris: Seuil, 1983-1985) y Alain Badiou, L'Être et l'Événement (Paris: Seuil, 1988).

${ }^{6}$ Véase por Ejemplo D. T. Starnes, "Purpose in the Writing of History", Modern Philology, vol. 20, 3 (1923): 281-300.

${ }^{7}$ Véase por ejemplo Henry Atlan, La science est-elle inhumaine? Essai sur la libre nécessité (Paris: Bayard, 2002) y Henry Atlan, Le Vivant Post-Génomique ou qu'est-ce que l'auto-organisation? (Paris: Odile Jacob, 2011).

${ }^{8}$ Véase por ejemplo Jorge Lozano, El discurso histórico (Madrid: Alianza, 1987).
} 
evolutiva no es una selección natural, sino artificial, determinada por medios tecnológicos. Más aún, ya no existe artificialidad alguna en este sentido por cuanto la naturalidad, el principio de lo natural que rige las sociedades contemporáneas, no puede ya basarse en los principios zoológicos y de filosofía naturalista del siglo XIX ${ }^{9}$ - evento que es aún más flagrante en zonas periféricas del planeta-. ${ }^{10}$

La relación entre lo natural y lo histórico ya no es evidente, sino discontinua y problemática, de manera que la construcción del presente en cuanto materia historiable debe adentrarse en áreas y dominios especulativos del todo inusitados para la historia clásica. Ya no habrá historia de individuos, de instituciones, de países, sino de aparatos, de conceptos, de condiciones territoriales, de imaginarios. Esta historia del presente será además siempre especulativa por cuanto no podrá ignorar las condiciones epistemológicas de su propia construcción.

Las teorías historiográficas, como reflexión última de lo histórico, serán reemplazadas por conceptos y aparatos, un área especulativa que podríamos por el momento llamar concepto paratografía, donde, para el analista de los hechos humanos, la dimensión específica de su tecnología no podrá distinguirse de la función cognitiva de la misma. Habrá entonces autores europeos como Bruno Latour (n. 1947) que centrarán su atención en los aparatos mismos y sus funcionamientos sociales; habrá otros, también europeos, como Bernard Stiegler (n. 1952), que se ocuparán del impacto cognitivo de este desarrollo tecnológico; y tendría que haber otros, de espacios periféricos, que se ocuparan de las nuevas formas coloniales que esta situación generará, no solo en relación a colonialismos ya establecidos -que recibirán sin duda otras formulaciones más sofisticadas- sino también en relación a nuevas e innovadoras maneras de colonialismo/colonización. En particular, a aquellas que, a falta de nuevo vocabulario, llamaremos por el momento colonialismo artificial, es decir, la existencia de un sistema de creciente colonialismo - cultural, financiero- no realizado a partir de una ideología, sino a partir de una situación diferencial de aparatos o máquinas y de su funcionamiento tecnológico. ${ }^{11}$ La novedad aquí reside en el hecho que esta situación diferencial existiría con independencia de toda lógica humana o corporativa, de modo que la intervención humana o corporativa, en algún momento y lugar del sistema o mundo, no permite extraer conclusiones analíticas acerca de por qué y cómo existen y evolucionan las formas coloniales.

Por otra parte, la idea de presente ya no es una cuestión acerca de cómo se determina el contenido del ahora y mientras tanto, sino acerca de qué -o a qué cosaconsideramos ahora y mientas tanto. De lo cual se deriva la discusión de lo terráqueo y lo poblacional, es decir, de cómo se constituye en términos cognitivos el espacio inmediato que llamamos mundo, casa, hogar, territorio, etc. y, sobre todo, acerca de cómo se halla amueblado y ocupado dicho mundo. Esto explica por qué hay historiadores que se interesan por ejemplo por problemas de zoología o por las teorías

\footnotetext{
${ }^{9}$ Véase por ejemplo Bernard Stiegler, La Technique et le Temps, tome 3: Le Temps du cinema et la Question du mal-être (Paris: Galilée, 2001), y Bernard Stiegler, Ce qui fait que la vie vaut la peine d'être vécue, de la pharmacologie (Paris: Flammarion, 2010).

${ }^{10}$ Véase Claudio Canaparo, El imaginario Patagonia (Bern: Peter Lang, 2011).

${ }^{11}$ Véase Claudio Canaparo, Geo-epistemology. Latin America and the Location of Knowledge (Oxford: Peter Lang, 2009) e Id., El imaginario.
} 
de Jacob von Uexküll (1864-1944) y Konrad Lorenz (1903-1989), u otros que encuentran en la etología de Boris Cyrulnik (n. 1937) una manera de explicar los comportamientos poblacionales recientes, u otros todavía que ven en las teorías del arquitecto de origen francés Bernard Tschumi (n. 1944) una forma de entender toda idea de organización territorial donde el quehacer humano es entendido principalmente como una cuestión de urbanismo y housing. Todo ello sin olvidar autores que a partir de dominios ya establecidos como la antropología o la logística analizan fenómenos extremos del pasado como los campos de concentración del nazismo. ${ }^{12}$

El ahora y mientras tanto adquiere su sentido de realidad inmediata a partir no de su naturaleza actual (de actualidad), como sucedía con los historiadores decimonónicos, sino de las construcciones en torno a expectativas y horizontes de espera de ese mismo ahora y mientras tanto. La condición de presente es así determinada por especulaciones y análisis en torno a proyecciones, teorías y estimaciones, que nunca se ocupan de lo que está sucediendo, sino de aquello que se cree puede suceder. Ningún significado relevante del presente tiene relación alguna con algo que solo sucede ahora y mientras tanto, sino con lo que se espera va a suceder y a partir de cual se establecen valores y significados de lo inmediato. Y es que la idea misma de que pueda especularse en torno al acontecer inmediato es algo que ha sido desmentido por autores tan disímiles como Paul Ricoeur y Hayden White (n. 1928). ${ }^{13}$

Aquí también se ha producido una confusión, frecuente sobre todo en las llamadas ciencias sociales, entre (i) el principio de asignación de realidad a ideas y conceptos, con (ii) el sentido material del mundo circundante (incluida su evolución y cambio) y con (iii) el estatuto del conocimiento (incluido el llamado conocimiento científico). El gran debate en torno a estas tres cuestiones ha residido menos en una perspectiva acerca de la historia que respecto del estatuto de lo conocible. Es decir, un presente para ser entendido como tal tiene que ser inteligible y para ello haber sido narrado o conceptualizado de alguna manera. ${ }^{14}$ Para otros autores, que sostienen una condición transparente y neutra de la escritura, esta situación de pasaje de no conocido a conocido no reviste un problema acerca del pasaje de lo que sucede (presente) a lo que ha sucedido (pasado entendido como tal). ${ }^{15}$ Para otros autores el asunto reside en la capacidad de significar lo que sucede y, por ende, la cuestión se halla en el pasaje (y en la elaboración de instrumento) de aquello que no tiene nombre hacia aquello que ya está conceptualizado. $^{16}$

\footnotetext{
${ }^{12}$ Véase por ejemplo Paz Moreno Feliú, En el corazón de la zona gris. Una lectura etnográfica de los campos de Auschwitz (Madrid: Trotta, 2010).

13 Véase por ejemplo Paul Ricoeur, Temps et récit (Paris: Seuil, 1983-1985) y Hayden White, Metahistory: The Historical Imagination in Nineteenth-Century Europe (Baltimore: The Johns Hopkins University Press, 1973), e Id., The Content of the Form: Narrative Discourse and Historical Representation (Baltimore: The Johns Hopkins University Press, 1987).

14 Véase por ejemplo Paul Veyne, Les Grecs ont-ils cru à leurs mythes? Essai sur l'imagination constituante (Paris: Éditions du Seuil, 1983).

15 John Ziman, Public Knowledge: Essay Concerning the Social Dimension of Science (Cambridge: Cambridge University Press, 1968), e Id., Real Science: What It Is and What It Means (Cambridge: Cambridge University Press, 2000).

${ }^{16}$ Véase por ejemplo Jacques Rancière, Les Noms de l'histoire. Essai de poétique du savoir (Paris: Le Seuil, 1992).
} 
Esta diversidad de situaciones permite no solo constatar la variedad polémica del panorama filosófico europeo, sino también el hecho de que las discusiones acerca de lo historiable no pasan por ninguna escuela específica de pensamiento reconocida por los historiadores -los "anales", historia de las mentalidades, etc.- sino por una preocupación que atraviesa a las ciencias sociales en su conjunto y al carácter de la actividad especulativa en general. De manera que las cuestiones epistemológicas que debieran interesar y orientar a los concernidos en lo historiable del mundo en que vivimos pasan menos por teorías en torno a diversas formas de definir lo histórico y mucho más en análisis acerca de los instrumentos disponibles para realizar lo historiable.

\section{Profile}

Claudio Canaparo es profesor visitante de Estudios Latinoamericanos del Birkbeck College de Londres (Reino Unido). Ha escrito como crítico literario, epistemólogo, analista de sociología de la cultura y filósofo. De sus últimas obras cabe destacar: $E l$ pensamiento basura. Transitoriedad, materia, viaje y mundo periférico (Peter Lang: Bern, 2017), El mundo Ingaramo (Peter Lang: Bern, 2015), El imaginario Patagonia. Ensayo acerca de la evolución conceptual del espacio (Peter Lang: Bern, 2011) y GeoEpistemology. Latin America and the Location of Knowledge (Peter Lang: Bern, 2009).

Claudio Canaparo is Visiting Professor in Latin American Studies at the Birkbeck College of London (United Kingdom). He has written as a literary critic, epistemologist, sociology of culture analyst, and philosopher. Of his most recent works stands out: $E l$ pensamiento basura. Transitoriedad, materia, viaje y mundo periférico (Peter Lang: Bern, 2017), El mundo Ingaramo (Peter Lang: Bern, 2015), El imaginario Patagonia. Ensayo acerca de la evolución conceptual del espacio, (Peter Lang: Bern, 2011) y GeoEpistemology. Latin America and the Location of Knowledge (Peter Lang: Bern, 2009.

Fecha de recepción: 15 de mayo de 2018.

Fecha de aceptación: 4 de junio de 2018.

Publicación: 30 de junio de 2018. 